\title{
Mathematical models for low-grade gliomas predict alternative radiotherapy fractionations with improved survival
}

\author{
Víctor M. Pérez-García ${ }^{1 a}$ \\ ${ }^{1}$ Instituto de Matemática Aplicada a la Ciencia y la Ingeniería and Departamento de Matemáticas, Universi- \\ dad de Castilla-La Mancha. Avda. Camilo José Cela 3, 13071 Ciudad Real, Spain.
}

\begin{abstract}
Low-grade gliomas are incurable infiltrative brain tumors that. In this abstract I summarize recent activity on a mathematical model of radiotherapy suggesting that alternative fractionations may led to a significantly better survival for this lethal disease.
\end{abstract}

\section{Introduction}

Gliomas are the most frequent type of primary brain tumors. Low grade gliomas (LGGs) are an incurable and very infiltrative subtype of primary brain tumors of astrocytic and/or oligodendroglial origin, with a median survival time around 5 years [1].

The therapeutical management of LGGs is based on a number of factors including age, performance status, location of tumor, and patient preference. Immediate radiotherapy after surgery increases the duration of response, but does not improve survival [2]. Thus, because of neurological toxicity, radiotherapy is usually offered only to patients with a combination of poor risk factors.

Mathematical modeling has the potential to help in selecting LGGs patients that may benefit from radiotherapy and in developing specific optimal fractionation schemes for selected patient subgroups.

Although many works have constructed mathematical models of high-grade gliomas there has been a limited number of works on low-grade gliomas. Ribba et al [3] developed an ODE-based model with a number of undetermined parameters that can be fit to describe patient's response with a good qualitative agreement. Also, Badoual et al. [4] have developed a simple spatially dependent mathematical model including the an odema dynamics that explains some observations of the volumetric evolution of low-grade gliomas [5].

In this abstract I will summarize our results on a mathematical model able to describe the known phenomenology of the response of LGGs to radiotherapy, and its application to develop novel treatment schemes [6-9].

\section{The Model}

\subsection{Tumor cell dynamics}

Glioma cell dynamics is described using the Fisher-Kolmogorov equation as in many other works [10]. Tumor cells, described as a density $u(x, t)$ measured in units of a maximal cell number, proliferate with a typical time $1 / \rho$ and have a characteristic mobility (diffusion) coefficient $D$.

\footnotetext{
ae-mail: victor.perezgarcia@uclm.es
} 
Cells that after the action of radiation are not able to repair the DNA damage feed a second compartment $v(x, t)$. These lethally damaged cells die after a time related to the typical proliferation time $1 / \rho$ and the average number of mitosis cycles $k$ that damaged cells are able to complete before dying. This is the so-called cell death through mitotic catastrophe, that is known to account for the majority of cell death after irratiation [11]. Thus the model reads

$$
\begin{aligned}
& \frac{\partial u}{\partial t}=D \Delta u+\rho(1-u-v) u, \\
& \frac{\partial v}{\partial t}=D \Delta v-\frac{\rho}{k}(1-u-v) v .
\end{aligned}
$$

on a domain $\Omega$ of the brain supplemented with initial data $u\left(x, t_{0}\right)=u_{0}(x), u_{0}(x) \in C^{2}(\bar{\Omega}), v\left(x, t_{0}\right)=0$, and no-flux boundary conditions $|\partial u / \partial \bar{n}|_{\partial \Omega}=0,|\partial v / \partial \bar{n}|_{\partial \Omega}=0$.

\subsection{Response to radiotherapy}

Radiotherapy is given in a very short time (minutes) in comparison with typical LGG evolution times (months), thus RT effect can be assumed to be instantaneous for the model timescales.

We take the damaged fraction of tumor cells to be given by the classical linear-quadratic model [11]. For a dose $d_{j}(\mathrm{~Gy})$ given at a time $t_{j}$, the fraction of cells that are not lethally damaged by a dose $d_{j}$ is given by $S_{j}=\exp \left(-\alpha_{t} d_{j}-\beta_{t} d_{j}^{2}\right)$, where $\alpha_{t}\left(\mathrm{~Gy}^{-1}\right)$ and $\beta_{t}\left(\mathrm{~Gy}^{-2}\right)$ are radiobiological constants.

The full treatment consists of a total dose $D$ split in a series of $n$ doses $d_{j}$ given at times $t_{j}$. In radiotherapy of LGGs the most frequently used scheme consists of $n=30$ doses of $d_{j}=1.8 \mathrm{~Gy}$, given from monday to friday during six weeks.

For a general radiation fractionation defined by the irradiation times $\left\{t_{j}\right\}_{j=1, \ldots, n}$ and doses per fraction $\left\{d_{j}\right\}_{j=1, \ldots, n}$ we get the matching conditions for the irradiation times $t_{j}$.

$$
\begin{aligned}
u\left(x, t_{j}^{+}\right) & =S_{j} u\left(x, t_{j}^{-}\right), \\
v\left(x, t_{j}^{+}\right) & =v\left(x, t_{j}^{-}\right)+\left[1-S_{j}\right] u\left(x, t_{j}^{-}\right), \quad \text { for } j \geq 1 .
\end{aligned}
$$

\subsection{Dealing with treatment toxicity}

The main limitation when using radiation therapy is the treatment toxicity. Damage to the normal tissue can be quantified for a given fractionation $\left(t_{j}, d_{j}\right)$ as (see e.g. [11] pp. 114).

$$
E_{h}=-\log \left[\prod_{j=1}^{n} S_{j}\right]=\alpha_{h}\left(D+\frac{1}{\alpha_{h} / \beta_{h}} \sum_{j=1}^{n} d_{j}^{2}\right) .
$$

where $\alpha_{h}, \beta_{h}$ are the radiobiological response parameters for normal tissue. Also, acute reactions such as radionecrosis depend on the maximal dose per fraction $d_{*}$ used. Thus Eq. (3) and $d_{j} \leq d_{*}$ are restrictions to be considered when designing optimal therapies on the basis of Eqs. (1) and (2).

\subsection{Optimal therapy design}

When designing optimal therapies we will account for the most harmful effect of the tumor: its potential to transform into a high-grade tumor. So the optimal therapy would be the one delaying the most the malignant transformation of the tumor. To do so one must keep the total cell density below a certain limit. Mathematically one would like to maximize the time $T$ that the maximal cell density is below a certain value $u_{*}$ that leads to the appearance of hipoxia and more malignant phenotypes as

$$
T: \max _{\Omega} u(t, x)+v(t, x) \leq u_{*}
$$




\section{Results}

\subsection{The model based on Eqs. (1) and (2) describes the response of LGGs to RT}

First, in Ref. [6, 7] we have studied the response of the mathematical model given by Eqs. (1) and (2) and have compared the outcome to the known clinical phenomenology.

Interestingly, the model predicts a response to RT that lasts for a very long time (years) even when the therapy effect is instantaneous, what explains the counterintuitive findings of Ref. [5]. Moreover, the effect is found to be dependent on the proliferation rate. Thus, the more aggresive tumors respond faster but regrow earlier than those less agressive, also in line with clinical observations [5]. This lead to the suggestion that a limited ammount of radiation, estimated to be between 10 and 15 Gy might be used to probe tumor aggressiveness without loss of effectivity in the treatment, and help in taking more informed therapeutical decisions.

Also, in agreement with clinical trials [2], the model predicts that RT can be defered with no loss of effectivity, thus avoiding early patient's toxicity.

\subsection{What is the best way to deliver 54 Gy of radiation in six weeks of treatment?}

Next, in Ref. [8], we posed the following question: if doses are to be given during six weeks as with the standard fractionation, what is the way of distributing the total dose that preserves the toxicity while making the time to malignant transformation as large as possible?

The result of the optimization problem solved using feasible direction methods was that the best schedulling is to give just $1.8 \mathrm{~Gy} /$ fraction as it is done in clinical practice.

\subsection{Protracted therapies may improve survival while maintaining toxicity}

Finally, in Ref. [9], we posed the question: If the dose per fraction is fixed to be 1.8 Gy as in current schemes, what is the dose interspacing leading to the best survival while maintaining the toxicity? In that limit the problem becomes technically simple and an explicit formula can be given for the optimal dose interspacing.

The dose interspacing found are between one and two months depending on the tumor growth rate. Faster-growing tumors require shorter dose inter-spacings. The predicted survival increase by this simple modification is between 1 and 2 years what may be significant specially for faster-growing LGGs.

\section{Experimental validation}

Although the predictions of the mathematical model developed in Refs. [6, 7] are very promising, it is not possible to test this kind of hypotheses in real patients without previous experimental validation.

Typical biomedical research involves the validation of ideas or concepts in-vitro and/or in animal models. However, because of their very slow growth, there are neither stablished cell lines nor animal models available for low-grade gliomas. This fact, together with the very long time period necessary to validate ideas in real patients has been a serious limitation for the development and implementation of novel ideas on therapies for LGGs.

However, it has been recently proven that certain high grade cell lines such as U251 have very slow growth when grown in microfluidic chips that recreates a three-dimensional setup with nutrients and oxigen gradients resembling physiological conditions [12]. These setups may allow testing the ideas once the appropriate scaling of radiosensitivity and proliferation rates are taken into account. 
Experiments are on the way to use those chips as test beds for the concepts arising in the mathematical models.

We hope that the concepts raised from the mathematical study may translate into useful applications for the clinical management of this lethal disease.

\section{References}

[1] N. Pouratian, D. Schiff. Management of low-grade glioma. Curr Neurol Neurosci Rep, 10, 224-31 (2010).

[2] Van den Bent MJ, Afra D, de Witte O, Ben Hassel M, Schraub S, Hoang-Xuan K, Malmström PO, Collette L, Piérart M, Mirimanoff R \& Karim AB. Long-term efficacy of early versus delayed radiotherapy for low-grade astrocytoma and oligodendroglioma in adults: the EORTC 22845 randomised trial, Lancet, 366, 985-990 (2005)

[3] Ribba B, Kaloshi G, Peyre M, Ricard D, Calvez V, Tod M, Cajavec-Bernard B, Idbaih A, Psimaras D, Dainese L, Pallud J, Cartalat-Carel S, Delattre JY, Honnorat J, Grenier E, Ducray F. A tumor growth inhibition model for low-grade glioma treated with chemotherapy or radiotherapy. Clin Cancer Res. 15, 5071-5080 (2012).

[4] Badoual M, Gerin C, Deroulers C, Grammaticos B, Llitjos J.-F., Oppenheim C, Varlet P. Oedemabased model for diffuse low-grade gliomas: application to clinical cases under radiotherapy. Cell. Prolif. 47 369-380 (2014).

[5] Pallud J, Llitjos JF, Dhermain F, Varlet P, Dezamis E, Devaux B, Souillard-Scemama R, Sanai N, Koziak M, Page P, Schlienger M, Daumas-Duport C, Meder JF, Oppenheim C \& Roux FX. Dynamic imaging response following radiation therapy predicts long-term outcomes for diffuse low-grade gliomas. Neuro-Oncology 14, 496-505 (2012).

[6] V. M. Pérez-García, M. Bogdanska, J. Belmonte, P. Shucht, L. Pérez-Romasanta. Delay effects in the response of low grade gliomas to radiotherapy: A mathematical model and its therapeutical implications. Mathematical Medicine and Biology 32, 307-329 (2015).

[7] L. A. Pérez-Romasanta, J. Belmonte-Beitia, A. Martínez-González, G. F. Calvo, V. M. PérezGarcía Mathematical model predicts response to radiotherapy of grade II gliomas, Reports of Practical Oncology and Radiotherapy, 18 S63 (2013).

[8] T. Galochkina, A. Bratus, V. M. Pérez-García Optimal radiation fractionation for low-grade gliomas: Insights from a mathematical model, Mathematical Biosciences 267 1-9 (2015).

[9] V. M. Pérez-García, L. Pérez-Romasanta Extreme protraction for low grade gliomas: Theoretical proof of concept of a novel therapeutical strategy Mathematical Medicine and Biology, doi:10.1093/imammb/dqv017

[10] Martirosyan NL, Rutter EM, Ramey WL, Kostelich EJ, Kuang Y, Preul MC. Mathematically modeling the biological properties of gliomas: A review. Math Biosci Eng. 12, 879-905 (2015).

[11] A. Van der Kogel, M. Joiner, Basic clinical radiobiology, Oxford University Press, 2009.

[12] Ayuso JM, Monge R, Llamazares GA, Moreno M, Agirregabiria M, Berganzo J, Doblaré M, Ochoa I and Fernández LJ, SU-8 based microdevices to study self-induced chemotaxis in 3D microenvironments. Front. Mater. 2:37 (2015). 